\title{
Mass transport to nanoelectrode arrays and limitations of the diffusion domain approach: theory and experiment.
}

Neus Godino ${ }^{l}$, Xavier Borrisé $e^{1,2}$, Francesc Xavier Muñoz ${ }^{l}$, Francisco Javier del Campo ${ }^{1 *}$ and Richard G. Compton ${ }^{3}$.

${ }^{1}$ Instituto de Microelectrónica de Barcelona, IMB-CNM-CSIC; Esfera UAB. Campus Universitat Autónoma de Barcelona. 08193 - Barcelona, Spain.

${ }^{2}$ Institut Català de Nanotecnologia, ICN-CIN2; Esfera UAB. Campus Universitat Autónoma de Barcelona; 08193 - Bellaterra. Barcelona, Spain.

${ }^{3}$ Department of Chemistry; Physical and Theoretical Chemistry Laboratory; University of Oxford; South Parks Road; OX1 3QZ - Oxford; United Kingdom

franciscojavier.delcampo@imb-cnm.csic.es; richard.compton@chem.ox.ac.uk

RECEIVED DATE (to be automatically inserted after your manuscript is accepted if required according to the journal that you are submitting your paper to)

CORRESPONDING AUTHOR FOOTNOTE. F. Javier del Campo. Tel.: +34-935.947.700; Fax number: +34-935.801.496. 
ABSTRACT. The diffusion domain approach is a general framework for the understanding, interpretation and prediction of the response of microelectrode arrays. This work exposes some of its limitations, particularly when dealing with nanoelectrode arrays of a few microns in size. This article provides an overview of the principles and assumptions underpinning the diffusion domain approach, and then applies it to the study of nanoelectrode arrays. The apparent disagreement between theory and experimental data, due to the importance of radial diffusion to nanoelectrode arrays compared to microelectrode arrays, is explained using simulations and experiments. The principle that an array of micro- or nano-electrodes eventually behaves as if the entire array were a single electrode of the size of the array, with its corresponding properties, applies always. However, while microelectrode arrays tend to behave as macroelectrodes, nanoelectrode arrays on the other hand may behave as microelectrodes. For the case of arrays of small numbers of electrodes, or array sizes of microns or less in size, this compromises one of the key assumptions of the diffusion domain approach, namely that inner electrodes in an array are equivalent, which may lead the unaware to erroneous conclusions.

KEYWORDS. Electrochemistry; microelectrode arrays; nanoelectrode arrays; Diffusion domain approach; voltammetry.

\section{MANUSCRIPT TEXT.}

\section{Introduction}

Nanoelectrodes and nanoelectrode arrays are the natural next step in electrode miniaturization after microelectrodes and their arrays. However, only recent advances in nanofabrication techniques and metrology have enabled the controlled fabrication of such devices. This, together with the fact that such techniques are very costly and not so widely available, has limited the number of publications to date in this field. Early reports date back to the late 1990's, but the field did not seem to boom until fifteen 
years later with the works of Arrigan et al. ${ }^{1-3}$, White ${ }^{4,5}$ and Murray ${ }^{6}$ who reported on the mass transport properties of nanoelectrodes of various geometries and their arrays.

One common and intriguing feature of the results presented in these works is the sigmoidal shape of the voltammograms recorded at nanoelectrode arrays even at moderate and low scan rates. This may seem striking if we draw an analogy between the mass transport behavior of nanoelectrode arrays and microelectrode arrays. Compton et al. have pointed to four main regimes of behavior affecting diffusional transport to microelectrode arrays. According to the relationship between size of the individual diffusion layer thicknesses, $\delta$, microelectrode size described by their radius, $r$, and the intercentre distance between microelectrodes, $\mathrm{d}^{7,8}$, and with increasing diffusion layer thickness, these four regimes are: (i) planar diffusion to each microelectrode as $\delta<<$ r, (ii) radial diffusion to each microelectrode as $\delta=r<d$, (iii) a transition zone when $r<\delta=<d$ and (iv) planar diffusion to the microelectrode array when $\delta>>>d$. Regime-ii is the optimum one and it corresponds to the case when the response of the whole array is equivalent to that of a single microdisk, times the number of microelectrodes integrating the array. Regime-iv, on the other hand, consists in a complete overlap of individual diffusion layers that results in the array behaving as an electrode of the size of the entire micro- or nano-electrode array. Hence for most microelectrode arrays steady-state currents are observed in regime-ii, and then as the diffusion layer grows the current tails off in regime-iv. However, nanoelectrode arrays are a special case because their size is typically a few microns, so they show steady-state currents corresponding to microelectrode behavior, regardless of the extent of overlap between adjacent diffusion layers.

This motivated us to study diffusion at nanoelectrode arrays in more detail, combining simulations and experiments with nanoelectrode arrays fabricated using electron-beam lithography ${ }^{9}$. We first attempted to simulate the behavior of nanoelectrode arrays using the diffusion domain approach that has been so helpful in the study of microelectrode arrays. However, the results cast by this approach conflicted with the empirical observations reported in the past and, as we will show here, also with our 
own experimental data.

Models of nanoelectrode arrays were built based on three-dimensional domains and the results compared with those from two-dimensional models and the available experimental data. The threedimensional simulations were in agreement with the experimental results, and showed that nanoelectrode arrays working under regime-iv behave as single microelectrodes under most conditions.

The main reason why a two-dimensional approach fails to correctly model the behavior of such nanoelectrode arrays is because it considers the particular case of a micro -or nano- electrode completely surrounded by other electrodes like itself, and ideally far from the edges of the array. In other words, the diffusion domain approach is best suited for large micro- and nanoelectrode arrays with significantly more microelectrodes in inner than in perimetric positions. This is partly why the diffusion domain approach fails to simulate the response of small nanoelectrode arrays in regime iv accurately. Perhaps another constraint should be added when dealing with small nanoelectrode arrays, regarding the size of the array vis-à-vis the size of the diffusion layer at the array. Since diffusion at microelectrodes is radial, supply of material to each nanoelectrode in an array of micrometric dimensions depends heavily on its position. Inner positions are characterised by being completely surrounded by neighbouring microelectrodes, and hence less material is able to reach them by diffusion compared to perimetric electrodes. Edge effects are magnified at small nanoelectrode arrays, where the hemispherical nature of the diffusion layer corresponding to the whole array is not accurately accounted for by this two-dimensional domain approach.

Therefore, in this work we alert of the risks entailed by the indiscriminate application of the diffusion domain approach to nanoelectrode arrays regardless of their size. We show that while the behavior of nanoelectrode arrays is to some extent analogous to that of microelectrode arrays, different approaches ought to be used to model them depending on their overall size. Our conclusion is that while the diffusion domain approach is a powerful technique, it can only be applied to nanoelectrode arrays occupying a large area, such as large ensembles of interdigitated nanoband electrodes or nanopatterned 
electrodes as the ones described in ${ }^{10}$.

\section{Experimental:}

\subsection{Chemicals and instrumentation}

$\mathrm{KCl}(99 \%)$ and $\mathrm{K}_{4} \mathrm{Fe}(\mathrm{CN})_{6} \cdot 3 \mathrm{H}_{2} \mathrm{O}(99 \%)$ were purchased from Sigma-Aldrich. They were ACS analytical grade and were used as received without any further purification. Solutions were prepared using pure de-ionized water $(18 \mathrm{M} \Omega \cdot \mathrm{cm})$. All electrochemical measurements were performed using a three-electrode configuration and a CHI700C bipotentiostat (CH Instruments, Texas, USA) connected to a PC. All electrodes were made from gold, A Pt ring was used as auxiliary electrode and a Metrohm $\mathrm{Ag} / \mathrm{AgCl}(3 \mathrm{M} \mathrm{KCl})$ was used as reference electrode. Nanoelectrode geometry was characterized by atomic force microscopy (AFM) 9, 11 using a Veeco Dimension 3100 (Veeco Instruments) in Tapping Mode.

\subsection{Fabrication of disk nanoelectrode arrays}

Nanoelectrode arrays were fabricated over gold microband electrodes that had previously been made using standard photolithographic techniques as described previously in ${ }^{12,}{ }^{13}$. Chips containing gold microbands were spin-coated with a 100nm layer of PMMA 950K. The exposure was done in a RAITH 150TWO. Conditions of exposure were: dose $=0.016 \mathrm{pC}$ for the $0.25 \mathrm{um}$ pitch dots and $0.02 \mathrm{pC}$ for the 1.5um pitch dots; accelerating voltage $=10 \mathrm{kV}$ and at $\mathrm{WD}=10 \mathrm{~mm}$. Development was carried out in a standard MIBK:IPA (1:3) solution during 30sec and stopped with IPA during 30sec.

\section{Theory}

The current response of micro- and nano-electrode arrays was simulated in two- and threedimensional domains using the commercial finite element software package Comsol Multiphysics 3.3 (COMSOL, SE) in combination with Matlab (The Mathworks, Inc.).

We simulated the one-electron reduction process of a species in solution, corresponding to: 


$$
O+e \underset{k b}{\stackrel{k f}{\rightleftharpoons} R}
$$

where $k_{f}$ and $k_{b}$ are heterogeneous rate constants defined by Butler-Volmer kinetics. ${ }^{14}$

$$
\begin{aligned}
& k_{f}=k_{s} e^{-\alpha F\left(E-E^{0^{\prime}}\right) / R T} \\
& k_{b}=k_{s} e^{(1-\alpha) F\left(E-E^{o^{\prime}}\right) / R T}
\end{aligned}
$$

$k_{s}$ is the standard kinetic rate constant and indicates the degree of a system lability. In the present case a value of $10^{-2} \mathrm{~ms}^{-1}$, representing a fast system, was used in the simulations. For the sake of comparison, experimental values are usually between $10^{-2}$ and $10^{-7} \mathrm{~ms}^{-1}{ }^{14,15} ; \alpha$ is the charge transfer coefficient assumed to be 0.5 in our simulations- ${ }^{16}, E^{o}$ is the formal potential of the redox couple, which we fixed at $0.25 \mathrm{~V}$ to facilitate later comparison with experimental data. Finally, $F$ is the Faraday constant, $R$ is the gas constant and $T$ is absolute temperature, set at $298 \mathrm{~K}$ in our simulations.

We wanted to emphasize the importance of electrode geometry on diffusional transport alone, and therefore convection and migration were disregarded in our simulations. Only Fickian diffusion towards the electrodes was considered according to:

$$
\frac{\partial c_{i}}{\partial t}=D_{i}\left(\frac{\partial^{2} c_{i}}{\partial x^{2}}+\frac{\partial^{2} c_{i}}{\partial y^{2}}+\frac{\partial^{2} c_{i}}{\partial z^{2}}\right)
$$

where $c$ is the concentration and $D$ the diffusion coefficient of the species in solution. The diffusion coefficient used in the models was $6.5 \cdot 10^{-10} \mathrm{~m}^{2} \mathrm{~s}^{-1}$, which corresponds to ferrocyanide in $0.1 \mathrm{M} \mathrm{KCl}^{14}$.

The convergence of our solutions was heavily influenced by the quality of the mesh. Therefore, for each set of boundary conditions, we progressively refined the mesh until a convergence better than $1 \%$ between two consecutive solutions was obtained. While this did not represent a particular problem in the case of two-dimensional domains, it turned the solution of three-dimensional cases extremely time consuming. Accuracy, on the other hand, was affected by the size of the domains, which we set large enough to ensure bulk-like conditions at the boundaries. Given the recessed nature of our nanoelectrode $\operatorname{arrays}^{2,3,17,18}$, made by electron beam lithography over a thin polymethyl-methylacrilate (PMMA) layer, 
the current was not compared with the expression for the steady-state current at an inlaid microdisk, namely $I=4 n F c D r$. Instead, we used the expression for recessed microelectrodes ${ }^{19}$ :

$$
I=\frac{4 n F C D r_{o}}{\left(\frac{4 L}{\pi r_{o}}+1\right)}(0.5)
$$

where $n$ is the number of transferred electrons, $r_{o}$ is the radius of the electrode, and $L$ is the value of the recess height.

\section{Results and Discussion}

\subsection{Nanoelectrode arrays and the diffusion domain approach}

It is known that the size and shape of the diffusion layer at an electrode array depends on several factors such as: (a) experimental timescale, (b) microelectrode geometry, (c) inter-electrode distance and (d) diffusion coefficient of the electroactive species involved ${ }^{7}$. The diffusion domain approach is a widely used technique to simulate disk microelectrode arrays ${ }^{7,8,20}$. It consists in assigning its own space to each microelectrode in an array, so that it is diffusionally independent from its neighbours, as outlined in Figure 1. The main advantage is that it enables the accurate simulation of diffusional transport to a three-dimensional array of microelectrodes solving the mass transport problem in a twodimensional domain which solution is then integrated around the $\mathrm{Z}$ axis to obtain the current at a single microdisk. Last, this current is multiplied by the number of electrodes in the array to obtain the total current.
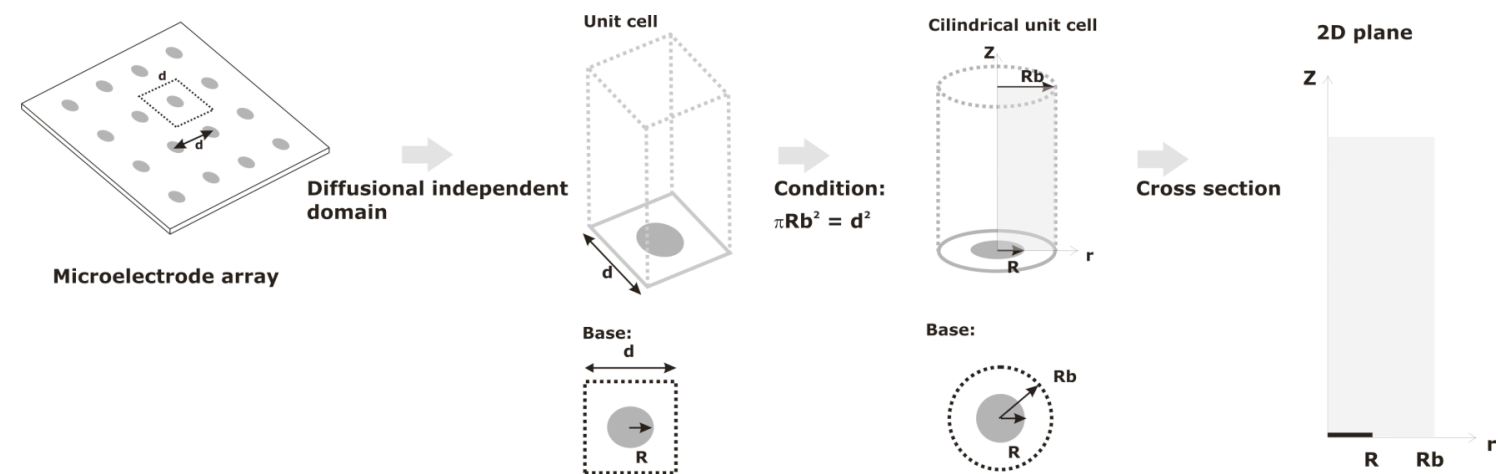

Figure 1 Schematic representation of diffusion domain approach. The final dimensional plane represents 
one microdisk electrode within an array of microdisks.

This approach works well both for regular ${ }^{7,8}$, and random arrays ${ }^{21}$ of microelectrodes as well as for partially blocked electrodes ${ }^{22,23}$. However, our results suggest that it is not suitable for the general simulation of nanometric-scale systems. In fact, this approach may be inaccurate in the case of disk nanoelectrode arrays where the footprint of the whole array is only a few microns square.

To show this, we used the diffusion domain approach ${ }^{20}$ and solved two-dimensional models for the reduction of species $O$, over square-lattice arrays of micro- and nano- disk electrodes. Figure 2A shows linear sweep voltammograms obtained for the case of a $5 \mu \mathrm{m}$ radius inlaid microelectrode separated by $100 \mu \mathrm{m}$ from its closest neighbors. The scan rates range from $5 \mathrm{mVs}^{-1}$ up to $1 \mathrm{Vs}^{-1}$. Figure $2 \mathrm{~B}$, on the other hand, shows the results for the case of a $50 \mathrm{~nm}$ radius nanodisk separated by $1 \mu \mathrm{m}$ from its immediate neighbors at scan rates going from $1 \mathrm{Vs}^{-1}$ up to $1000 \mathrm{Vs}^{-1}$. For fixed solution composition and centre-to-centre distance in the array, the degree of overlapping of the diffusion layers mainly depends on the scan rate used ${ }^{7,8}$.

(A)

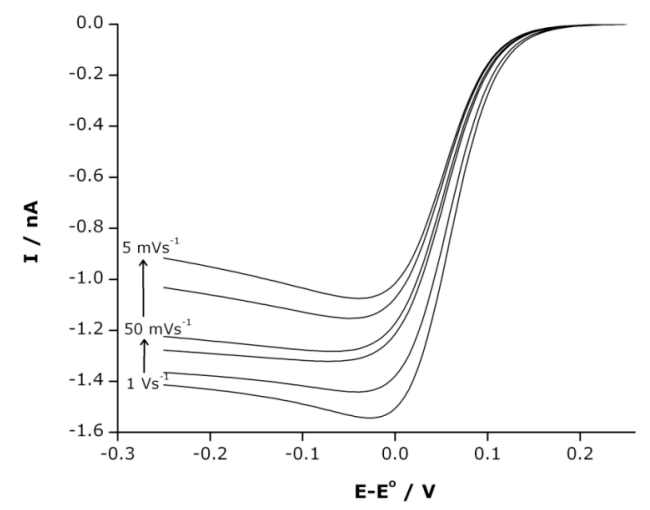

(B)

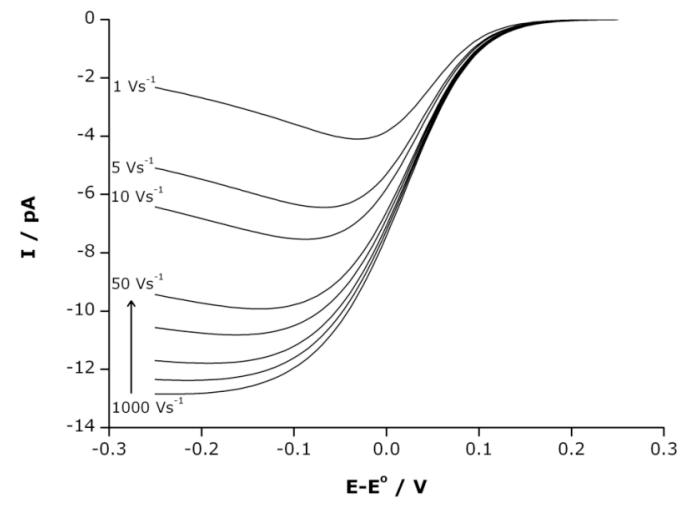

Figure 2 Simulated linear sweep voltammograms at different disk electrodes within an array, using the diffusion domain approach. (A) Microdisk radius of $5 \mu \mathrm{m}$ and an inter-center distance of $100 \mu \mathrm{m}$ for $0.005,0.01,0.05,0.1,0.5$ and $1 \mathrm{Vs}^{-1}$. (B) Nanodisk radius of $50 \mathrm{~nm}$ and an inter-center distance of $1 \mu \mathrm{m}$ 
for $1,5,10,50,100,250,500$ and $1000 \mathrm{Vs}^{-1}$. In both cases $E^{o^{\prime}}=0.25 \mathrm{~V}, D=6.5 \cdot 10^{-10} \mathrm{~m}^{2} \mathrm{~s}^{-1}$ and $[\mathrm{O}]=1 \mathrm{mM}$.

Our simulation results for the microelectrode array (Figure 2A) agreed with previous results presented in ${ }^{8}$. The transition across the different regimes can be seen in the figure, and further confirmation may be obtained using Einstein's relation $\delta=\sqrt{2 D t}=\sqrt{2 D \Delta E / v}$, where a value of $0.150 \mathrm{~V}$ may be used for $\Delta E$, and $v$ is the scan rate in $\mathrm{Vs}^{-1}$. Thus, at scan rates faster than $1 \mathrm{Vs}^{-1}$ the voltammograms show a peak associated to planar diffusion governing mass transport to the microelectrodes (Figure 2A). As the scan rate decreases, diffusion becomes hemispherical. Around $50 \mathrm{mVs}^{-1}$ the microelectrodes still behave independently from each other and the current recorded by the array corresponds to the limiting current of a single microelectrode times the number of elements in the array. As the scan rate continues to decrease, the degree of overlapping between diffusion layers increases and eventually the array behaves as a macroelectrode of the same area as that occupied by the array.

In the case of nanoelectrode arrays the diffusion domain approach casts a similar landscape, and it would seem that the only difference is that, for the nanoelectrodes in the array to be diffusionally independent, faster scan rates would be required (50 $\mathrm{Vs}^{-1}$ or higher in our example). This seems perfectly normal, but it does not fit with the available experimental results reported by several authors ${ }^{1-}$ 3, 24-26. These works show clearly sigmoidal voltammograms obtained at moderate-to-low scan rates using nanoelectrode arrays.

We believe that when the electrode geometry shifts from the micro- into the nano-scale, all other parameters -diffusion coefficients, concentrations and experiment duration- being the same, special attention needs to be paid to the concentration profiles across the diffusion layer of the whole array. This means that voltammetric peaks might be observed for species with sufficiently low diffusion coefficients, such as ionic liquids, but also for nanoelectrode arrays occupying an area of millimeter dimensions.

The four different behavior cases of microelectrode arrays, which depend on the overlapping of the 
individual diffusion layers, were presented in ${ }^{7,8}$. Usually, the different regimes may be reached by changing either the inter-electrode distance or the duration of the experiment for a given analyte. In the case of microelectrode arrays, which occupy areas in the order of a few square millimetres, the diffusion domain approach works because the consumption of material is large enough to change the concentration profiles very significantly in the vicinity of the array. Nanoelectrodes on the other hand, present a much smaller area and hence the amount of material that they are able to electrolyse is also very small to affect the solution composition to a great extent, even if they operate long enough to result in the complete overlap of their diffusion layers -at least so long as the footprint of the array is of micrometric dimensions!-. In fact, the sigmoidal voltammograms reported in the literature ${ }^{1-3,24-26}$ appear almost independent of scan rate.

To test these ideas we performed another kind of two-dimensional simulations. We modeled a sectional plane containing the central row of electrodes in a 10x10 array. Again, the domain was drawn large enough to avoid misleading results due to the proximity of the boundaries and the mesh was duly refined. Figure 3 shows close-up images of the concentration maps in the vicinity of the micro- (3A) and the nano-electrode array (3B). They are clearly different. Figure 3A corresponds to a microelectrode array sweeping the potential at $5 \mathrm{mVs}^{-1}$; the diffusion layers of the individual electrodes clearly overlap, and diffusion to the array is nearly planar. This is within reasonable agreement with the diffusion domain approach, which predicts a planar diffusion layer under these conditions.

On the other hand, the concentration map for the nanoelectrode array sweeping at $1 \mathrm{Vs}^{-1}$, shown in Figure 3B, suggests that the diffusion domain approach, which according to Figure $2 \mathrm{~B}$ pointed to mildly planar diffusion, was inaccurate. Figure 3B shows that the diffusion layer for the array, where the diffusion layers of the individual nanoelectrodes are completely overlapped, is actually hemispherical ${ }^{6}$. We believe that this is why the data shown in Figure 2B and the experimental results presented in bibliography ${ }^{1-3,24-26}$ differ. The implication is that while the domains of each nanoelectrode may be diffusionally independent, it would be wrong to assume that these nanoelectrodes are equivalent. This is 
particularly so for arrays occupying an area of micrometric dimensions, where the consumption on the part of the nanoelectrodes is so little that the solution bulk can readily supply fresh material to the diffusion layer so that the concentration in the vicinity of the array remains very close to the bulk value. Therefore, the contribution of radial diffusion to nanoelectrode arrays is more important than it is of microelectrode arrays ${ }^{6}$.

We believe that very large nanoelectrode arrays would be required for the diffusion domain approach to predict the behavior of the system as accurately as in the case of microelectrode arrays. However, a system like that may be treated as a partially blocked electrode rather than as an array of nanoelectrodes.

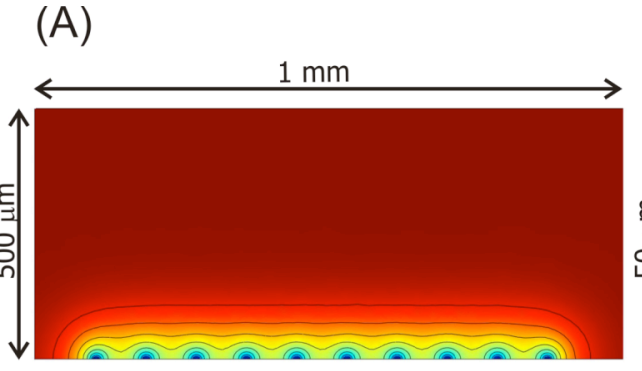

10 electrodes

$\mathrm{r}=5 \mu \mathrm{m}$ $\mathrm{d}=100 \mu \mathrm{m}$
(B)

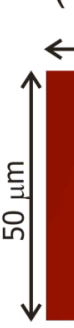

concentration
$\mathrm{mol} \cdot \mathrm{m}^{-3}$

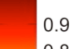

0.8

0.7
0.6

0.5

0.4

0.3

Figure 3 Details from 2D simulations of a plane perpendicular to a row of disk electrodes within an array of 10x10 electrodes. (A) 10x10 microdisks of $5 \mu \mathrm{m}$ of radius at $100 \mathrm{~s}$ (r) and $100 \mu \mathrm{m}$ of intercenter distance at $0.5 \mathrm{~s}$ (d) (B) 10x10 nanodisks of $50 \mathrm{~nm}$ of radius and $1 \mu \mathrm{m}$ of intercenter distance. In both cases $[\mathrm{O}]=1 \mathrm{mM}$ and $\mathrm{D}=6.5 \cdot 10^{-10} \mathrm{~m}^{2} \mathrm{~s}^{-1}$.

\subsection{D Modelling of recessed-disk nanoelectrode arrays}

It seems that the consumption of electroactive species by the nanoelectrodes is so small that diffusion from the bulk quickly replenishes the consumed material. The result is that the nanoelectrodes in the array are no longer equivalent and reducing the system dimensions from 3D to $2 \mathrm{D}$ for the sake of modeling becomes inadequate. Radial contribution is so high in these systems that it is necessary to introduce 3D-domain models because the diffusion domain approach cannot simulate these devices 
accurately. Therefore we built three-dimensional models of nanoelectrode arrays and used them to study the effect, on the system response, of scan rate, nanoelectrode separation and nanoelectrode population. In all cases, the nanoelectrodes were disks of $50 \mathrm{~nm}$ radius that were recessed $100 \mathrm{~nm}$ in an insulating material. We chose these values because they are easy to achieve using standard nanofabrication techniques such as electron beam lithography (EBL) or focused ion beam (FIB) ${ }^{9}$.

In all cases, the diffusion coefficient used was $6.5 \cdot 10^{-10} \mathrm{~m}^{2} \mathrm{~s}^{-1}$, corresponding to ferrocyanide in $0.1 \mathrm{M}$ $\mathrm{KCl}^{14}$. The simulated concentration of species $O$ was $20 \mathrm{mM}$ in all cases to facilitate direct comparison with experimental data.

\subsubsection{Effect of scan rate}

Figure 4 shows the results of two 6x6 nanoelectrode arrays with different intercenter distances, $250 \mathrm{~nm}$ and $1.5 \mu \mathrm{m}$, for scan rates ranging from 0.1 up to $5000 \mathrm{Vs}^{-1}$. As the scan rate increased, the resulting currents for both distances seemed to converge. In other words, the nanoelectrodes in the array were gradually becoming diffusionally independent and hence closer to being equivalent to each other. The effect was more manifest with decreasing inter-nanoelectrode distance because overlapping of neighboring diffusion layers at low scan rates was more significant.

It is important to point out that for scan rates below $1 \mathrm{Vs}^{-1}$, where overlap of adjacent diffusion layers was complete, the current still presented stationary values and the voltammograms were sigmoidal, as shown in Figure 5. This result is in agreement with the experimental observations from other authors ${ }^{1-3}$, ${ }^{24-26}$, and differs from the typical behavior that a microelectrode array would show. That is, when the diffusion layers of neighboring microelectrodes in an array overlap completely, the behavior of the array becomes that of a macroelectrode and the voltammograms tail off due to planar diffusion. The low scan rate behavior of nanoelectrode arrays, on the other hand, resembles that of a microelectrode. 


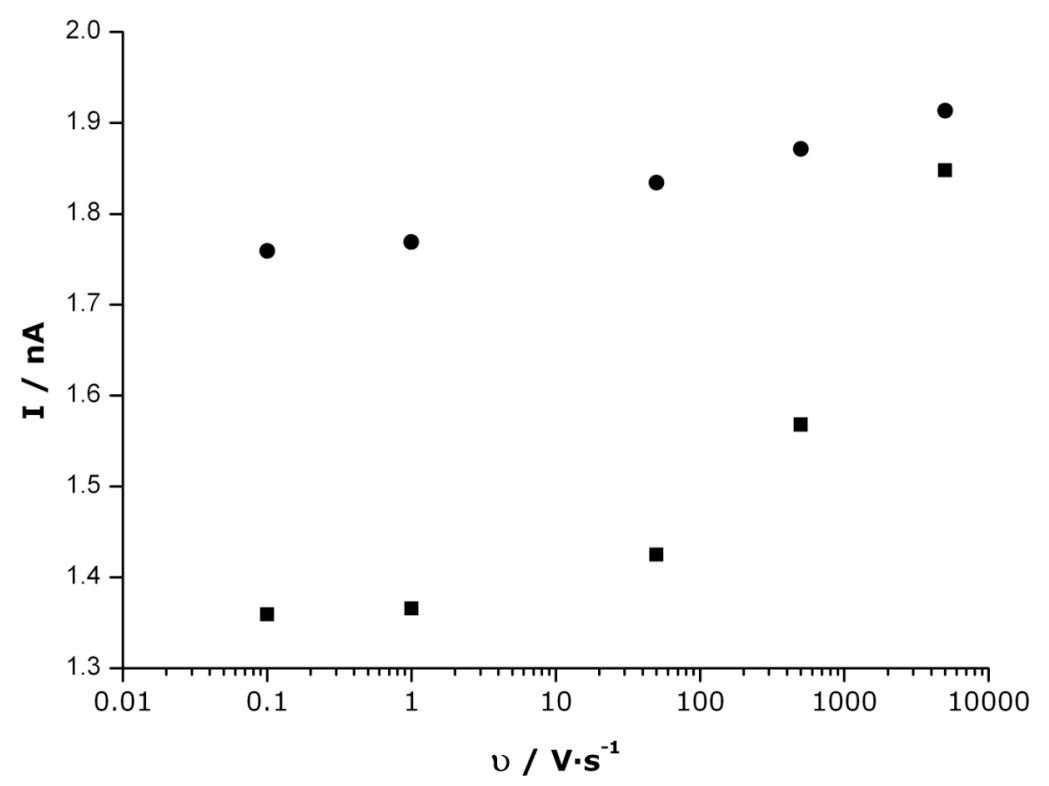

Figure 4 Simulation results for steady state current at $\left(E-E^{\circ}\right)=-0.25 V$ vs scan rate for a $6 \times 6$ nanodisks array of $50 \mathrm{~nm}$ of radius, $100 \mathrm{~nm}$ of recess and an intercenter distance of $1.5 \mu \mathrm{m}(\bullet)$ and $0.250 \mu \mathrm{m}(\boldsymbol{\bullet})$. In both cases $[\mathrm{O}]=20 \mathrm{mM}$ and $\mathrm{D}=6.5 \cdot 10^{-10} \mathrm{~m}^{2} \mathrm{~s}^{-1}$. A logarithmic axis is used in order to represent a wide range of scan rates.

\subsubsection{Effect of nanoelectrode separation}

The second parameter studied was the centre-to-centre distance between nanoelectrodes. The simulated results for a $6 \times 6$ nanoelectrode array at $1 \mathrm{Vs}^{-1}$ are showed in Figure 6 . As the intercenter distance increases the steady state current is close to the theoretical current, equation (0.5), multiplied by the number of electrodes in the array (36 electrodes). This raises questions regarding the practical aspects of nanoelectrode array design: is it really necessary to ensure diffusional independence between nanoelectrodes? And, if so, are those nanoelectrode arrays still true nanometric devices? Taking our 6x6 nanoelectrode array, if diffusional independence was to be achieved at a scan rate of approximately $1 \mathrm{Vs}$ ${ }^{1}$, then the nanoelectrodes would have to be separated at least $3 \mu \mathrm{m}$ from each other -about 30 times their size!-. This would lead us to a device 18 x $18 \mu \mathrm{m}$, equivalent to an approximately 10 micron radius microelectrode, except for the fact that the current at the 10 micron radius electrode would be higher. 
Under these circumstances, nanoelectrode arrays should contribute with other advantages such as decreased capacitive currents and the possibility to work in even more resistive media than a conventional microelectrode, which might be of interest in fast voltammetry or in-vivo studies.

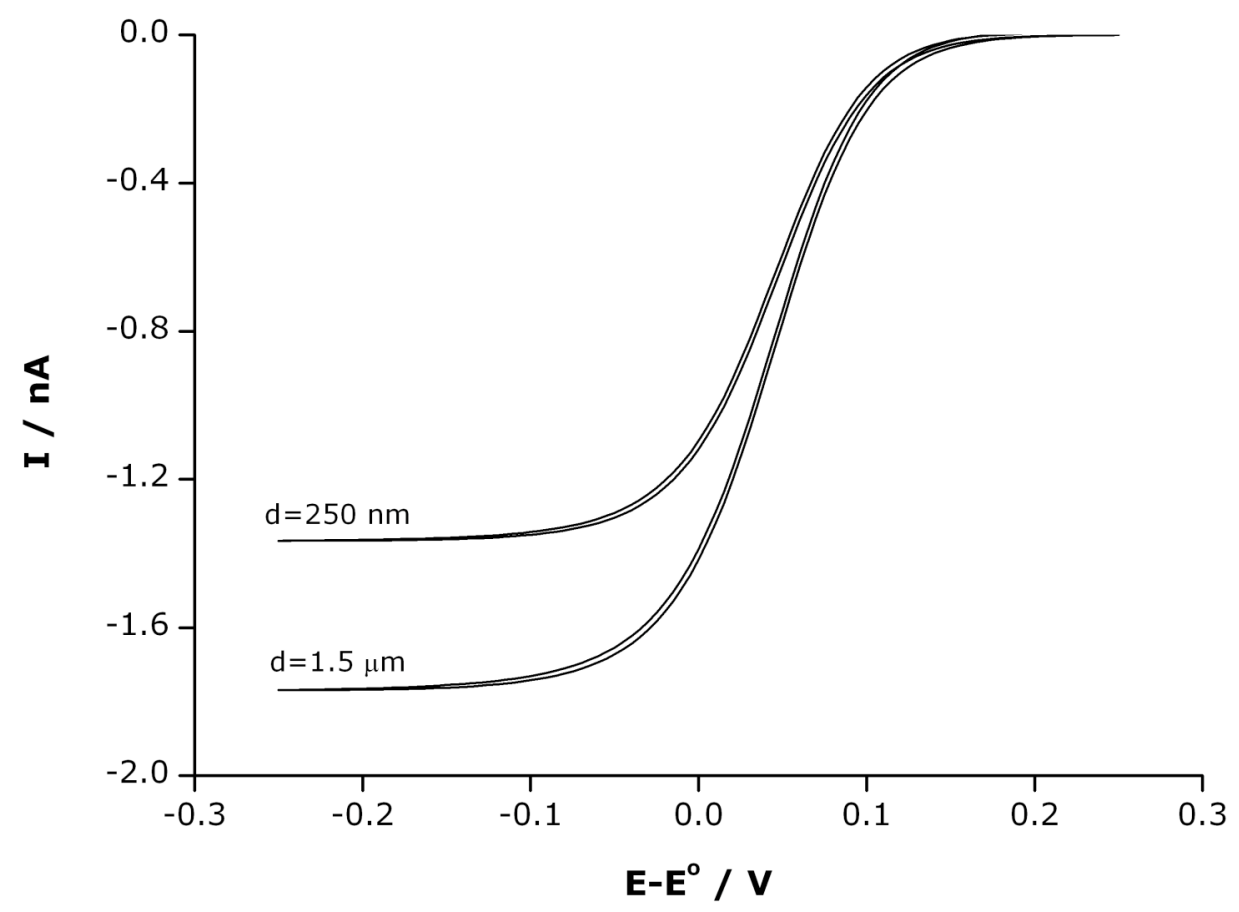

Figure 5 Simulated voltamograms at $1 \mathrm{Vs}^{-1}$ for a $6 \times 6$ nanodisks array of $50 \mathrm{~nm}$ of radius, $100 \mathrm{~nm}$ of recess and two intercenter distances $(\mathrm{d}): 1.5 \mu \mathrm{m}$ and $0.250 \mu \mathrm{m}$. In both cases $[\mathrm{O}]=20 \mathrm{mM}$ and $\mathrm{D}=6.5 \cdot 10^{-}$ ${ }^{10} \mathrm{~m}^{2} \mathrm{~s}^{-1}$ 


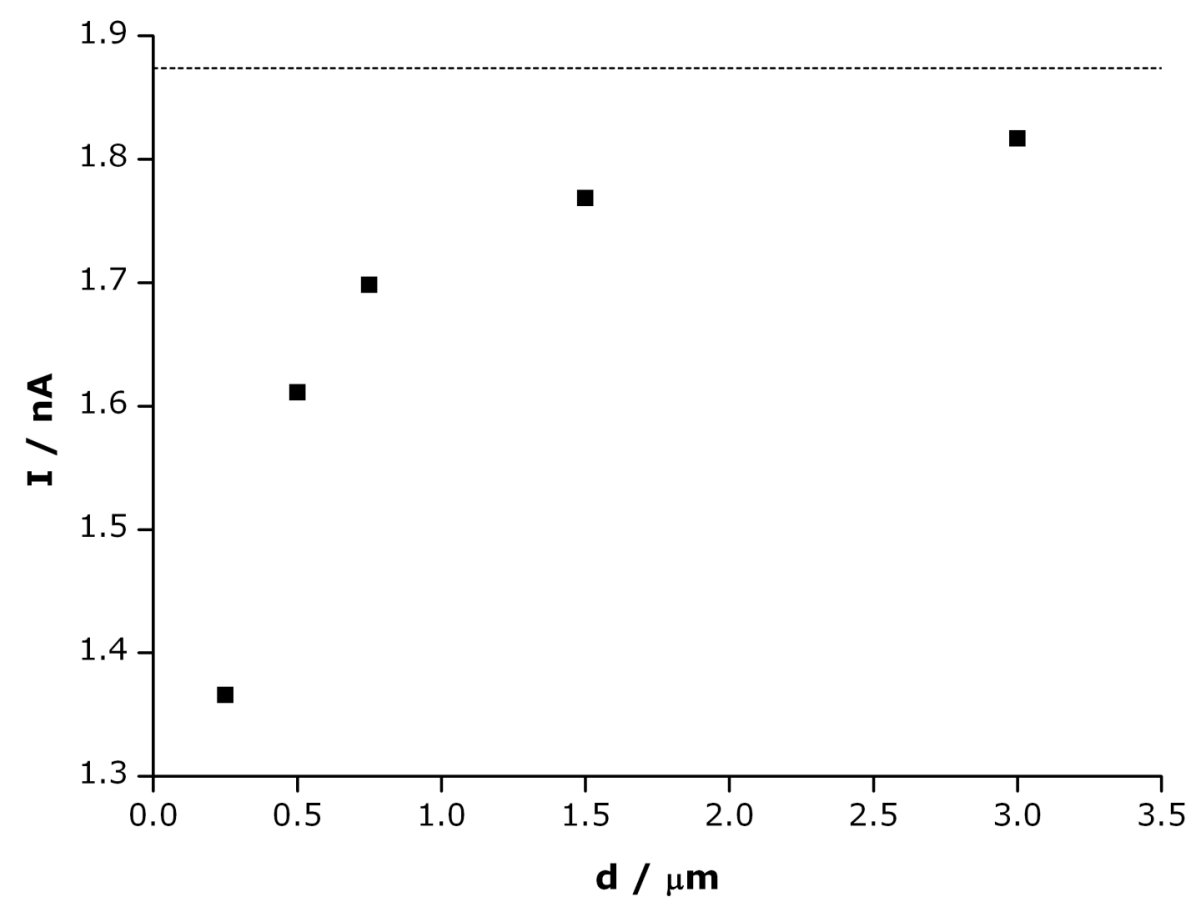

Figure 6 Simulation results for steady state current at $\left(E-E^{0}\right)=-0.25 \mathrm{~V}$ and $1 \mathrm{Vs}^{-1}$ vs intercenter distance (d) for a $6 \times 6$ nanodisks array of $50 \mathrm{~nm}$ of radius and $100 \mathrm{~nm}$ of recess. In both cases [O] $=20 \mathrm{mM}$ and $\mathrm{D}=6.5 \cdot 10^{-10} \mathrm{~m}^{2} \mathrm{~s}^{-1}$. The logarithmic axis is used in order to represent a wide range of scan rates. The dotted line represents the theoretically expected current at an array of diffusionally independent nanoelectrodes as per equation (0.5).

\subsubsection{Effect of nanoelectrode population}

Finally, we considered nanoelectrode population as the last parameter affecting the behavior of nanoelectrode arrays. Figure 7 shows the results for three different nanoelectrode arrays $(3 \times 3,6 \times 6$ and 9x9) for scan rates ranging between 0.1 and $5000 \mathrm{Vs}^{-1}$. For the sake of comparison, the simulated steady-state currents were normalized with respect to the number of electrodes in each array. These normalized currents were regarded as the average current observed by each nanoelectrode in the array. This is only an approximation because, as we showed earlier, the nanoelectrodes in an array are far from equivalent. Figure 7 also depicts, as a dotted line, the theoretical current for an infinitely isolated 
nanoelectrode of identical geometry, based on equation 0.5. It was found that the normalized current approached the theoretical value for the isolated electrode as the scan rate increased, independently from the number of electrodes integrating the array. These were obvious results that reminded of the behavior of microelectrode arrays. As the scan rate increases, the degree of overlap between adjacent diffusion layers decreases and the current recorded by each nanoelectrode in the array approaches the theoretical response of a single nanoelectrode, regardless of the population size of the nanoelectrode array. At slow scan rates, on the other hand, the average current was found to depend heavily on the number of the nanoelectrodes in the array. The simulations showed that as the nanoelectrode population in an array increased, the average current per nanoelectrode decreased. This is also in line with the behavior observed at microelectrode arrays; as the number of electrodes in an array increases, there are more electrodes at inner positions competing for the diffusing material. These inner positions are actually diffusionally equivalent and the ones that we believe are successfully modelled by the diffusion domain approximation. The situation is different in the case of those electrodes located around the edges of the array. Not being completely surrounded by other electrodes, they face less competition for the diffusing species and hence they experience higher current densities than inner electrodes. The trend to observe less and less current (per nanoelectrode) as nanoelectrode population in the array increases also has a limit. Hypothetically, this limit is reached when the nanoelectrodes inside the array far outnumber perimetric ones. 


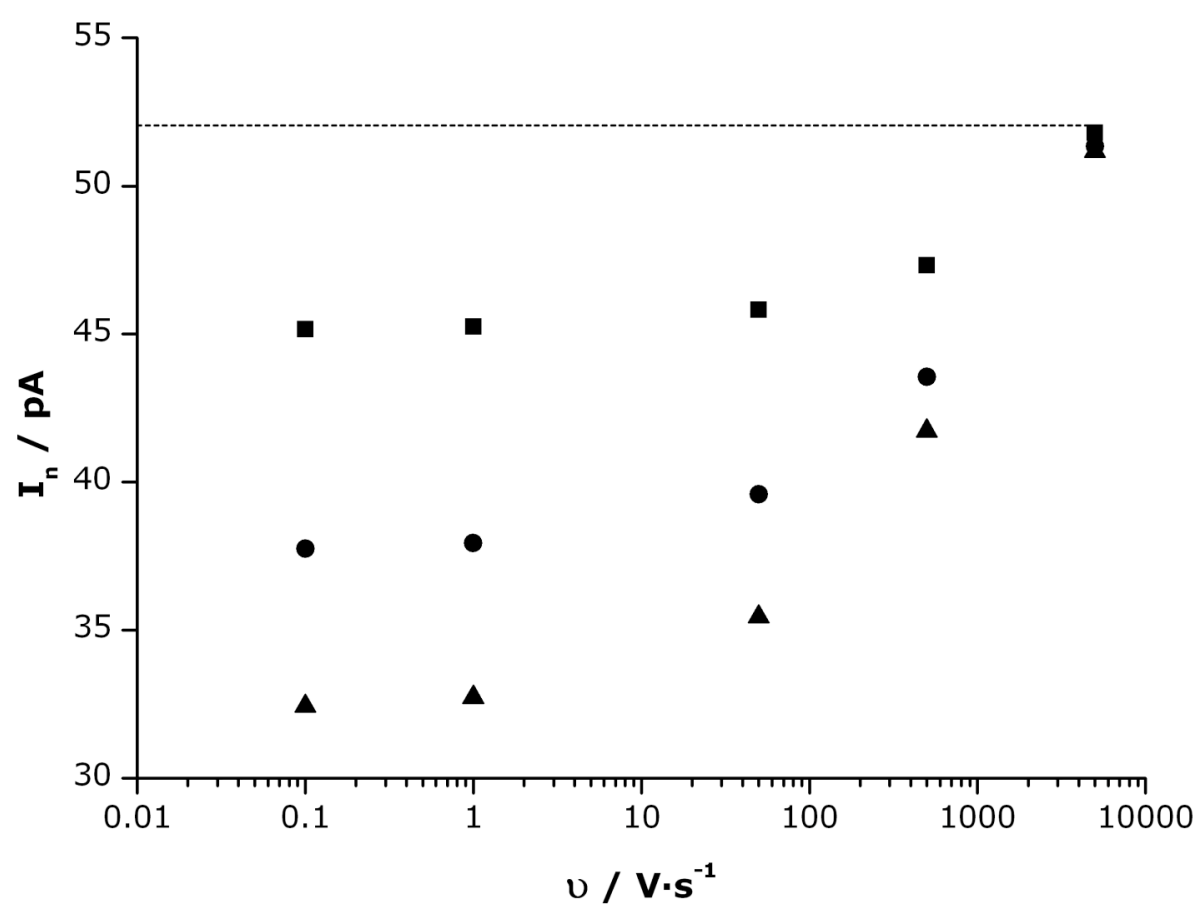

Figure 7 Simulation results for normalized steady state current at $\left(\mathrm{E}^{-} \mathrm{E}^{\circ}\right)=-0.25 \mathrm{~V}$ vs scan rate for a $3 \times 3$ nanodisks array $(\bullet), 6 \times 6$ nanodisks array $(\bullet)$ and 9x9 nanodisks array $(\boldsymbol{\Delta})$. It is normalized to the total number of electrodes per array. In all cases $\mathrm{r}=50 \mathrm{~nm}, \mathrm{~L}=100 \mathrm{~nm}, \mathrm{~d}=250 \mathrm{~nm},[\mathrm{O}]=20 \mathrm{mM}$ and $\mathrm{D}=6.5 \cdot 10^{-10} \mathrm{~m}^{2} \mathrm{~s}^{-1}$.

\subsection{Experimental results}

The three kinds of nanoelectrode arrays described earlier in section 2.2 were used in cyclic voltammetry experiments to study their mass transport properties compared to microelectrode arrays. Also, the experimental results were of great help to validate our three-dimensional models. Nanoelectrode arrays presenting different inter-nanoelectrode separations and nanoelectrode populations were produced, and Table 1 provides a summary of their main geometric features, measured using by AFM. Figure 8 shows AFM pictures from two arrays presenting inter-nanoelectrode separations of 1.5 $\mu \mathrm{m}$ and $0.250 \mu \mathrm{m}$, respectively. 

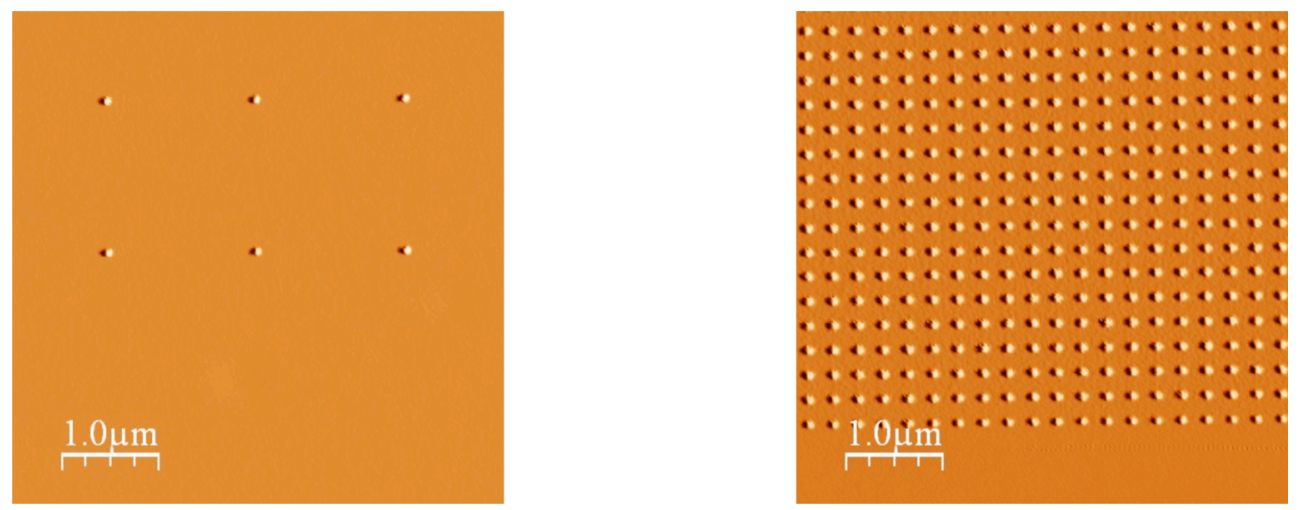

Figure 8 AFM image of an array of (A) $1.5 \mu \mathrm{m}$ and (B) $250 \mathrm{~nm}$ of intercenter distance.

Table 1 Geometric features for each array configuration. Errors are calculated based on 10 measurements.

\begin{tabular}{|l|l|l|l|}
\hline Number of electrodes & Diameter $/ \mathrm{nm}$ & Recess height/ $\mathrm{nm}$ & Intercenter distance \\
\hline $6 \times 6$ array & $114 \pm 8$ & $152 \pm 5$ & $1.5 \mu \mathrm{m}$ \\
\hline $31 \times 31$ array & $133 \pm 8$ & $156 \pm 10$ & $250 \mathrm{~nm}$ \\
\hline $61 \times 61$ array & $180 \pm 14$ & $73 \pm 6$ & $250 \mathrm{~nm}$ \\
\hline
\end{tabular}

Although the nanoelectrodes described in table 1 present important differences in their size and recess height, they were of the same magnitude and could be qualitatively compared with the simulated currents reported in the previous section.

Figure 9 shows the voltamograms at $100 \mathrm{mVs}^{-1}$ for each array in a $20 \mathrm{mM}$ ferrocyanide solution. It is remarkable that all voltamograms are sigmoidal and reach a steady-state current. This is so even for the densely populated arrays $\mathrm{B}$ and $\mathrm{C}$, where the diffusion layers of the individual nanoelectrodes are certainly overlapping, and the number of inner nanoelectrodes (3721) is far greater than that of perimetric (240) nanoelectrodes for the $61 \times 61$ array. This clearly points to the importance of radial diffusion to these devices, likely explained by the overall size of the array, which occupies a $15 \times 15$ 
micron square area. This is roughly the area of a microelectrode of 8.5-micron radius.

(A)

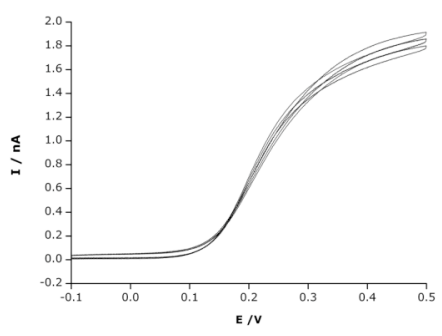

(B)

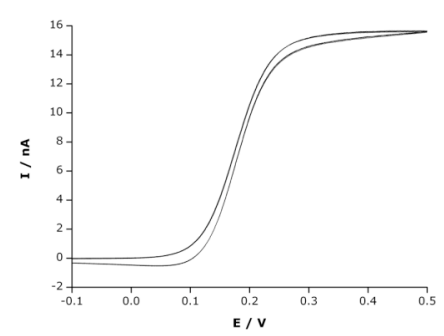

(C)

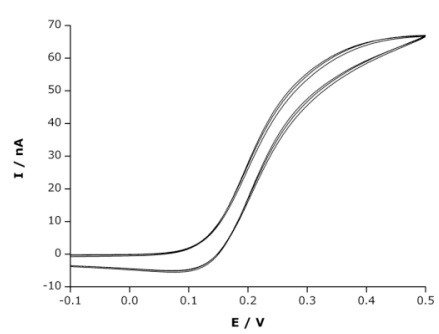

Figure 9 Experimental voltamogragrams for each array configuration at $100 \mathrm{mVs}^{-1}$ in $20 \mathrm{mM}$ ferrocyanide / 0.1 M KCl solutions (A) 6x6 nanoarray, (B) 31x31 nanoarray and (C) 61x61 nanoarray. Three measurements are shown to demonstrate reproducibility.

We then took the data obtained from the $6 \times 6$ nanoelectrode array and compared them to our simulations. The other two arrays were too large and their simulation was abandoned because we were unable to solve models of that size.

Figure 4 shows the average steady-state currents per electrode, measured at different scan rates for a 3D-modelled 6x6 nanoelectrode array with an intercenter distance of $1.5 \mu \mathrm{m}$. At $100 \mathrm{mVs}^{-1}$ the simulated current was $1.76 \mathrm{nA}$, which compared very favorably with an experimental value of $1.7 \pm 0.1$ $\mathrm{nA}$ at the same scan rate. This experimental value was obtained from triplicate measurements conducted at five different nanoelectrode arrays of this kind.

Figure 10 shows the experimental steady-state currents for the other two nanoarray configurations. Again, the data have been normalized with respect to the number of electrodes in each array. The figure shows that at higher scan rates the two sets of data deviate from each other, as each is affected to a different extent by capacitive currents. The more nanoelectrodes in the array, the higher its capacitive currents will be. This was sistematically found when comparing the $61 \times 61$ array to the $31 \times 31$, even at the lowest scan rates. This can also be seen in figures $9 \mathrm{C}$ and $9 \mathrm{~B}$. Nevertheless, when we compared 
Figure 10 and Figure 7 we reached the following conclusions. First, the normalized current values at low scan rates are very similar in both arrays, around $15 \mathrm{nA}$ and, as expected, they are also considerably lower than the values obtained for the simulated arrays of 3x3, 6x6 and 9x9 (Figure 7). The coincidence in average current per nanoelectrode found for arrays $61 \times 61$ and $31 \times 31$ can be explained by the fact that in both cases there are many more inner nanoelectrodes than perimetric ones.

At higher scan rates, the shape of the experimental results followed the same trend as predicted by the simulations. This means that, as the scan rate increases, the individual diffusion layers overlap less and less until they eventually become independent from each other (Figure 7), and this upper limit may be estimated using equation $(0.5)$.

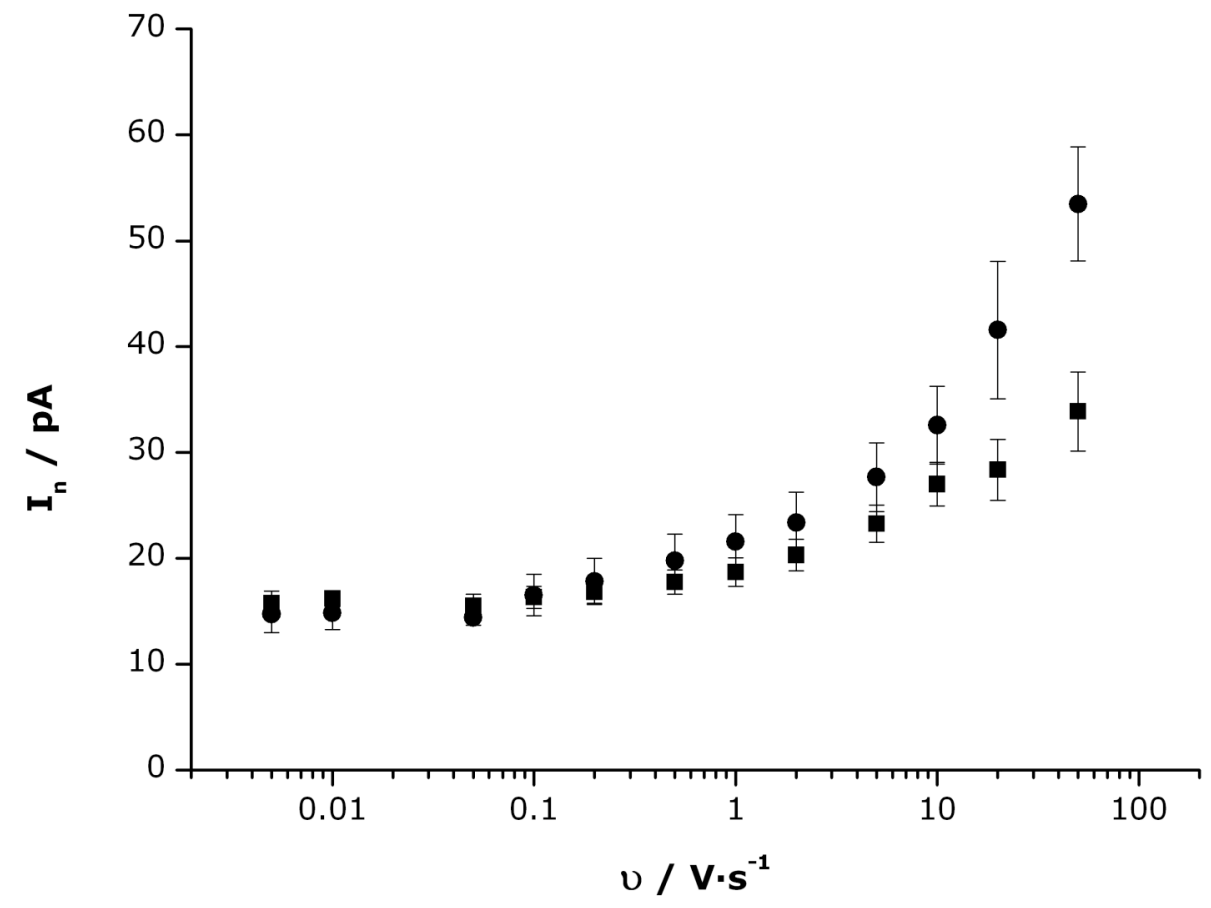

Figure 10 Experimental results for normalized steady state current at $\mathrm{E}=0 \mathrm{~V}$ ss scan rate for a $31 \mathrm{x} 31$ nanodisks array $(\bullet)$ and 61x61 nanodisks array $(\bullet)$. It is normalized to the total number of electrodes per array. Concentration of ferrocyanide used is $20 \mathrm{mM}$. Error bars are calculated using 4 different nanoarrays for each configuration and measurements were done in triplicate for each scan rate. 
Our experimental results are in high concordance with previous results published by several authors ${ }^{1-}$ ${ }^{3,24-26}$. In all these works the behavior for the their nanoelectrode arrays was sigmoidal, even working at quite low scan rates. Furthermore, our experimental results also corroborated the modeled results described in the previous section.

\section{Conclusions}

We have shown that diffusion to nanoelectrode arrays depends very much on the size of the array. Thus, nanoelectrode arrays occupying areas of a few micron square observe steady state even if the diffusion layers of adjacent nanoelectrodes overlap completely. In this case, the magnitude of the observed current is expected to be slightly lower than that experienced by a microelectrode of comparable dimensions, which experiences radial diffusion when $D t>r^{2}$.

This has important implications related to the design and characterisation of this kind of nanoelectrode-based devices. For example, theoretical paradigms that are valid to explain the behavior of microelectrode arrays and nanopatterned macroelectrodes are very likely unsuitable for the study of nanoelectrode arrays. We have shown this using several two- and three-dimensional models together with recessed-nanoelectrode arrays fabricated by electron beam lithography.

We have shown the effect of inter-nanoelectrode spacing and also nanoelectrode population in different regular arrays arranged in a square lattice. The currents observed at nanoelectrode arrays seem to have an upper and a lower limit. Drawing an analogy to microelectrode arrays, the upper limit is reached at short operation times and corresponds to regimes i and ii. The lower current limit is reached for regime-iv, when the diffusion layers of adjacent nanoelectrodes are completely overlapped and the array effectively behaves as a microelectrode. This means that radial diffusion to nanoelectrode arrays is more important than in microelectrode arrays, and hence theoretical approaches that were valid for the latter may be no longer applicable to nanoelectrode arrays of micrometric size. If this is the case then new theoretical approaches are needed to model these devices and improve our understanding of them. 
ACKNOWLEDGEMENT. NG acknowledges a PhD grant from the Spanish Ministry of Science and Innovation (MICINN) JDC gratefully acknowledges a Ramón y Cajal Fellowship also from MICINN.

\section{REFERENCES}

(1) Arrigan, D. W. M. Analyst 2004, 129, 1157-1165.

(2) Lanyon, Y. H.; Arrigan, D. W. M. Sensors and Actuators, B: Chemical 2007, 121, 341-347.

(3) Lanyon, Y. H.; De Marzi, G.; Watson, Y. E.; Quinn, A. J.; Gleeson, J. P.; Redmond, G.; Arrigan, D. W. M. Anal. Chem. 2007, 79, 3048-3055.

(4) Zhang, B.; Zhang, Y.; White, H. S. Anal. Chem. 2004, 76, 6229-6238.

(5) Zhang, B.; Zhang, Y.; White, H. S. Anal. Chem. 2006, 78.

(6) Murray, R. W. Chem.Rev. 2008, 108, 2688-2720.

(7) Davies, T. J.; Compton, R. G. J. Electroanal. Chem. 2005, 585, 63-82.

(8) Davies, T. J.; Ward-Jones, S.; Banks, C. E.; del Campo, J.; Mas, R.; Munoz, F. X.; Compton, R. G. J. Electroanal. Chem. 2005, 585, 51-62.

(9) Bushan, B., Ed. Handbook of Nanotechnology, 2nd ed., 2007.

(10) Compton, R. G.; Wildgoose, G. G.; Rees, N. V.; Streeter, I.; Baron, R. Chem. Phys. Lett. 2008, 459, 1-17.

(11) Horcas, I.; Fernández, R.; Gómez-Rodríguez, J. M.; Colchero, J.; Gómez-Herrero, J.; Baro, A. M. Rev. Sci. Instrum. 2007, 78.

(12) Ordeig, O.; Banks, C. E.; Davies, T. J.; del Campo, F. J.; Munoz, F. X.; Compton, R. G. Anal. 
Sci. 2006, 22, 679-683.

(13) Ordeig, O.; Banks, C. E.; Davies, T. J.; del Campo, J.; Mas, R.; Munoz, F. X.; Compton, R. G. Analyst 2006, 131, 440-445.

(14) Allen J. Bard, L. R. F. ELECTROCHEMICAL METHODS. Fundamentals and Applications, 2nd ed.; John Wiley \& sons: New York, 2001.

(15) Scholz, F., Ed. Electroanalytical Methods: Guide to Experiments and Applications; Springer, 2002.

(16) Wang, J. Analytical Electrochemistry, Second Edition ed.; Wiley-VCH, 2000.

(17) Bartlett, P. N.; Taylor, S. L. J. Electroanal. Chem. 1998, 453, 49-60.

(18) Galceran, J.; Taylor, S. L.; Bartlett, P. N. J. Electroanal. Chem. 1999, 476, 132-147.

(19) Bond, A. M.; Luscombe, D.; Oldham, K. B.; Zoski, C. G. J. Electroanal. Chem. 1988, 249, 1-14.

(20) Amatore, C.; Saveant, J. M.; Tessier, D. J. Electroanal. Chem. 1983, 147, 39-51.

(21) Ordeig, O.; Banks, C. E.; Davies, T. J.; del Campo, J.; Munoz, F. X.; Compton, R. G. J. Electroanal. Chem. 2006, 592, 126-130.

(22) Davies, T. J.; Banks, C. E.; Compton, R. G. J. Solid State Electrochem. 2005, 9, 797-808.

(23) Davies, T. J.; Moore, R. R.; Banks, C. E.; Compton, R. G. J. Electroanal. Chem. 2004, 574, 123 152.

(24) Baker, W. S.; Crooks, R. M. J. Phys. Chem. B 1998, 102, 10041-10046.

(25) Ito, T.; Audi, A. A.; Dible, G. P. Anal. Chem. 2006, 78, 7048-7053.

(26) Sandison, M. E.; Cooper, J. M. Lab on a Chip - Miniaturisation for Chemistry and Biology 
2006, 6, 1020-1025. 